\title{
Desenvolvimento de mudas de baru em resposta a diferentes volumes de tubetes e doses de adubo de liberação lenta
}

\author{
Marcos Vinícius Miranda Aguilar ${ }^{1 *}$, Marília Dutra Massad ${ }^{2}$, Tiago Reis Dutra ${ }^{3}$, Fabiano Guimarães Silva ${ }^{4}$, \\ Eduarda Soares Menezes ${ }^{5}$, Aline Ramalho dos Santos ${ }^{6}$
}

DOI: https://doi.org/10.35699/2447-6218.2020.25629

\begin{abstract}
Resumo
O presente trabalho objetivou avaliar a produção e a qualidade de mudas de baru em resposta a diferentes volumes de tubetes e aplicação de doses de Osmocote ${ }^{\circledR}$. Foi adotado o delineamento experimental em blocos casualizados, com três repetições, no esquema fatorial 3 x 5, sendo estudados três volumes de tubetes $\left(55,180\right.$ e $\left.280 \mathrm{~cm}^{3}\right)$ e cinco doses de Osmocote ${ }^{\circledR}\left(0 ; 3,0 ; 6,0 ; 9,0\right.$ e 12,0 $\left.\mathrm{g} \mathrm{dm}^{-3}\right)$, do Osmocote ${ }^{\circledR}$ MiniPrill Controlled Realise 19-06-10, com liberação de 3 a 4 meses. Cada unidade experimental foi constituída por 10 mudas. Foram avaliados aos 120 dias a altura $(\mathrm{H} ; \mathrm{cm})$, diâmetro do coleto (DC, mm), matéria seca da parte aérea (MSPA; g planta ${ }^{-1}$ ), matéria seca da raiz (MSR; $g$ planta ${ }^{-1}$ ) e matéria seca total (MST = MSPA + MSR; $g$ planta-1 ${ }^{-1}$, além das relações H/DC, H/MSPA, MSPA/MSR e Índice de Qualidade de Dickson (IQD). O tubete de $280 \mathrm{~cm}^{3}$ proporcionou maior crescimento inicial e produção de massa seca às mudas de Dipteryx alata (Vogel), conferindo um padrão de qualidade superior. As mudas de baru responderam ao uso do adubo de liberação lenta Osmocote ${ }^{\circledR}$, apresentando melhores crescimento e padrão de qualidade sob doses entre 4,35 e $5,29 \mathrm{~g} \mathrm{dm}^{-3}$.
\end{abstract}

Palavras-chave: Dipteryx alata (Vogel). Osmocote ${ }^{\circledR}$. Produção de mudas. Qualidade de mudas. Silvicultura.

\section{Development of baru seedlings in response to different tube volumes and slow release fertilizer doses}

\author{
Abstract

\footnotetext{
${ }^{1}$ Universidade Federal de Santa Maria. Santa Maria, RS. Brasil.

https://orcid.org/0000-0003-0480-6119

${ }^{2}$ Instituto Federal do Norte de Minas Gerais. Salinas, MG. Brasil.

https://orcid.org/0000-0002-2599-0157

${ }^{3}$ Instituto Federal do Norte de Minas Gerais. Salinas, MG. Brasil.

https://orcid.org/0000-0002-8991-1454

${ }^{4}$ Instituto Federal do Norte de Minas Gerais. Salinas, MG. Brasil.

https://orcid.org/0000-0002-9367-8958

${ }^{5}$ Universidade Federal dos Vales do Jequitinhonha e Mucuri. Diamantina, MG. Brasil. https://orcid.org/0000-0003-1476-5402

${ }^{6}$ Universidade Federal do Espírito Santo. Jerônimo Monteiro, ES. Brasil. https://orcid.org/0000-0002-1560-3555

*Autor para correspondência: aguilarmarcos2009@hotmail.com
}

The present work aimed to evaluate the production and quality of baru seedlings in response to different volumes of tubes and application of doses of Osmocote ${ }^{\circledR}$. A randomized block design was used, with three replications, in a $3 \mathrm{x}$ 5 factorial scheme, with three volumes of tubes $\left(55,180\right.$ and $\left.280 \mathrm{~cm}^{3}\right)$ and five doses of Osmocote ${ }^{\circledR}(0 ; 3.0 ; 6.0 ; 9.0$ and $12.0 \mathrm{~g} \mathrm{dm}^{-3}$ ), of Osmocote ${ }^{\circledR}$ MiniPrill Controlled Perform 19-06-10, with release of 3 to 4 months. Each experimental unit was verified by 10 seedlings. At 120 days height $(\mathrm{H} ; \mathrm{cm})$, stem diameter (DC, $\mathrm{mm})$, shoot dry matter

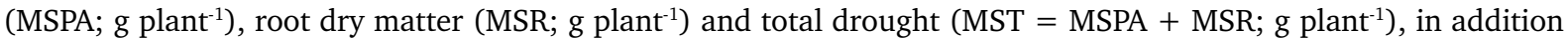

Recebido para publicação em 01 de outubro de 2020. Aceito para publicação em 19 de novembro de 2020 . e-ISSN: 2447-6218 / ISSN: 2447-6218. Atribuição CC BY. 
Aguilar, M. V. M. et al.

to the H / DC, H / MSPA, MSPA / MSR and Dickson Quality Index (IQD) ratios. The $280 \mathrm{~cm}^{3}$ tube provided greater initial growth and dry mass production for the Dipteryx alata (Vogel) seedlings, providing a superior quality standard. The baru seedlings responded to the use of Osmocote ${ }^{\circledR}$ slow-release fertilizer, the best growth and quality standard at doses between 4.35 and $5.29 \mathrm{~g} \mathrm{dm}^{-3}$.

Key words: Dipteryx alata (Vogel). Osmocote ${ }^{\circledR}$. Seedling production. Seedling quality. Forestry.

\section{Introdução}

O Cerrado brasileiro compreende uma das maiores savanas do mundo, e com sua grande variabilidade de habitat é considerado um dos principais hotspots de diversidade (Sousa et al., 2018). No entanto, o crescimento populacional alarmante, aliado a expansão agrícola e a alta demanda por produtos madeireiros, aumentou a exploração das florestas nativas, resultando em grandes taxas de desmatamento, que culminaram na degradação de extensas áreas, em virtude do seu abandono após a perda da capacidade produtiva ou manejo incorreto do solo (Silva et al., 2020).

A revegetação dessas áreas é fundamental para melhorar as características físicas e químicas do solo, fornecendo, por meio da cobertura vegetal, a proteção necessária para diminuir os processos erosivos (Freitas et al., 2017). Assim, surge a necessidade da produção de mudas de espécies nativas para compensar essas perdas, e reestabelecer as condições ecológicas do ambiente (Santos et al., 2018).

Dentre as espécies potenciais para o reflorestamento, podemos citar a Dipteryx alata (Vogel), conhecida popularmente como baru, nativa do Bioma Cerrado, pertence a família Fabaceae e subfamília Faboideae. É indicada para a recuperação de áreas degradadas, devido ao seu rápido crescimento, alta produção de massa foliar, baixa exigência de adubação e de manutenção (Silva et al., 2020). É considerada promissora para o cultivo, devido ao seu uso múltiplo, alta taxa de germinação e estabelecimento de mudas. Apresenta potencial na indústria de medicamentos e na alimentação humana e animal, já que a polpa e as sementes são energéticas, nutritivas e ricas em minerais (Guimarães et al., 2019).

A diversidade de produtos e serviços ofertados pelas espécies florestais, aliada à preocupação mundial com relação ao meio ambiente, tem promovido um aumento na demanda por mudas nativas (Menegatti et al., 2017). Dessa forma, torna-se necessário o desenvolvimento de protocolos e estratégias, que favoreçam a produção das mesmas com qualidade, em menor custo e tempo, possibilitando assim atender aos objetivos dos plantios (Massad et al., 2017).

Dentre os fatores que exercem influência sobre a qualidade, e os custos de produção de mudas de espécie florestais pode-se destacar o volume do recipiente e as demandas nutricionais das espécies (Dias et al., 2018).
O tamanho do recipiente para a produção de mudas em viveiros florestais tem influência direta em seu custo final, pois deste resulta a quantidade de substrato a ser utilizado, o espaço que ocupado no viveiro, a mão de obra utilizada no transporte, remoções para aclimatação e retirada para entrega ao produtor, além da influência na quantidade de insumos e/ou fertilizantes e água que irá demandar (Lima Filho et al., 2019).

O equilíbrio entre o volume do tubo e a qualidade das mudas pode ser gerenciado com o suprimento adequado de nutrientes através da fertilização (Cabreira et al., 2019). Uma alternativa para esse fim é a aplicação de fertilizantes de liberação controlada, como o Osmocote ${ }^{\circledR}$, que promove uma utilização mais eficiente dos nutrientes, menor taxa de lixiviação e volatilização (Garibay et al., 2018).

O Osmocote ${ }^{\circledR}$ é um fertilizante que libera nutrientes de maneira controlada (Gibson et al., 2019). É formado por cápsulas com nutrientes envoltos por uma resina orgânica biodegradável, que assegura proteção, contudo disponíveis por pressão osmótica, a fim de permitir a liberação lenta do nutriente ativo, mantendo a liberação prolongada de nutrientes em sincronia com as necessidades metabólicas da planta (Santos et al., 2020).

As práticas de adubação favorecem o desenvolvimento das mudas, e dessa forma diminui o tempo de permanência das plantas no viveiro, reduzindo assim os custos de produção (Brito et al., 2018). A aplicação de fertilizantes que possuem liberação lenta ou ação controlada de nutrientes permite uma disponibilidade contínua do mesmo, evitando o uso parcelado de algumas fontes, e diminuindo os custos de operação (Gomes et al., 2020).

Otimizar o uso dos recursos para produção de mudas no viveiro se mostra importante, principalmente para as empresas florestais, que necessitam produzir grandes quantidades de mudas em menor tempo, visando baixo custo e padrão de qualidade (Massad et al., 2017). Entretanto, estudos que avaliem diferentes volumes de recipientes, e o efeito de adubos de liberação lenta em espécies nativas ainda são incipientes. Assim, torna-se necessário ampliar as pesquisas sobre a reposta das espécies nativas à diferentes doses de Osmocote ${ }^{\circledR} \mathrm{e}$ volumes de tubetes, a fim de se determinar as formas de manejo das mudas. 
Desenvolvimento de mudas de baru em resposta a diferentes volumes de tubetes e doses de adubo de liberação lenta

Desta forma, o presente trabalho objetivou avaliar a produção e a qualidade de mudas de baru em resposta a diferentes volumes de tubetes e aplicação de doses de Osmocote ${ }^{\circledR}$.

\section{Material e Métodos}

\section{Área de estudo}

O trabalho foi conduzido no "Viveiro de Produção de Mudas Florestais" do Instituto Federal de Educação, Ciência e Tecnologia do Norte de Minas Gerais (IFNMG), Campus Salinas. O município de Salinas encontra-se localizado entre $16^{\circ} 10^{\prime} 19^{\prime \prime}$ de latitude Sul, e $42^{\circ} 17^{\prime} 30^{\prime \prime}$ de longitude Oeste, e está situado na mesorregião do Norte de Minas possuindo altitude média de $471 \mathrm{~m}$. O clima da região, de modo geral, varia de subúmido a semiárido, classificado majoritariamente, segundo Köppen, como sendo Aw, de inverno seco e verão chuvoso (Toledo et al., 2009).

\section{Condução do experimento}

Foi adotado o delineamento experimental em blocos casualizados, com três repetições, no esquema fatorial $3 \times 5$, sendo estudados três volumes de tubetes $\left(55,180\right.$ e $\left.280 \mathrm{~cm}^{3}\right)$ e cinco doses de Osmocote ${ }^{\circledR}(0 ; 3,0$; 6,0; 9,0 e 12,0 $\mathrm{g} \mathrm{dm}^{-3}$ ), do Osmocote ${ }^{\circledR}$ MiniPrill Controlled Realise 19-06-10, com liberação de 3 a 4 meses. Cada unidade experimental foi constituída por 10 mudas.

O substrato utilizado para produção das mudas foi a base de casca de Pinus moída (Rohrbacher ${ }^{\circledR}$ ), cuja caracterização química foi realizada conforme descrito por Empresa Brasileira de Pesquisa Agropecuária (EMBRAPA, 1997). As características físicas de porosidade total, macroporosidade, microporosidade, e capacidade máxima de retenção de água dos substratos foram determinadas por metodologia proposta por Carvalho e Silva (1992) (Tabela 1).

Tabela 1 - Características químicas e físicas do substrato utilizado na produção de mudas de baru

\begin{tabular}{|c|c|}
\hline \multirow{2}{*}{ Características $^{1}$} & Substrato \\
\hline & Rohrbacher ${ }^{\circledR}$ \\
\hline $\mathrm{pH}$, água & 6,0 \\
\hline M.O, dag kg-1 & 15,49 \\
\hline $\mathrm{P}, \mathrm{mg} \mathrm{dm}{ }^{-3}$ & 262 \\
\hline $\mathrm{K}, \mathrm{mg} \mathrm{dm}^{-3}$ & 968 \\
\hline $\mathrm{Ca}, \mathrm{cmol}_{\mathrm{c}} \mathrm{d} \mathrm{m}^{-3}$ & 5,07 \\
\hline $\mathrm{Mg}, \mathrm{cmol}_{\mathrm{c}} \mathrm{d} \mathrm{m}^{-3}$ & 1,59 \\
\hline $\mathrm{Al}, \mathrm{cmol}_{\mathrm{c}} \mathrm{d} \mathrm{m}^{-3}$ & 0,00 \\
\hline $\mathrm{H}+\mathrm{Al}, \mathrm{cmol}_{\mathrm{c}} \mathrm{d} \mathrm{m}^{-3}$ & 2,17 \\
\hline $\mathrm{t}, \mathrm{cmol}_{\mathrm{c}} \mathrm{d} \mathrm{m}^{-3}$ & 9,19 \\
\hline $\mathrm{T}, \mathrm{cmol}_{\mathrm{c}} \mathrm{d} \mathrm{m}^{-3}$ & 11,38 \\
\hline $\mathrm{SB}, \mathrm{cmol}_{\mathrm{c}} \mathrm{d} \mathrm{m}^{-3}$ & 9,19 \\
\hline $\mathrm{m}, \%$ & 0 \\
\hline $\mathrm{V}, \%$ & 82 \\
\hline Porosidade Total, \% & 62,26 \\
\hline Macroporosidade, \% & 30,99 \\
\hline Microporosidade, \% & 31,27 \\
\hline CMRA, mL $55 \mathrm{~cm}^{-3}$ & 24,52 \\
\hline
\end{tabular}

1 M.O. = matéria orgânica; $\mathrm{t}$ = capacidade efetiva de troca de cátions; $\mathrm{T}$ = capacidade de troca de cátions; $\mathrm{SB}=$ soma de bases; $\mathrm{m}=$ saturação por alumínio; $\mathrm{V}$ = saturação por bases; CMRA = Capacidade máxima de retenção de água.

As sementes de baru (Dipteryx alata (Vogel)) foram coletadas de dez árvores matrizes localizadas no município de Riachinho-MG. Para superar a impermeabi- lidade do tegumento das sementes de baru, foi realizada a quebra da dormência por meio do método da escarificação mecânica utilizando lixa d'água número 60 até 
Aguilar, M. V. M. et al.

desgastar o tegumento no lado oposto ao da micrópila, conforme Dutra et al., 2013.

Posteriormente, as sementes foram desinfestadas em solução de hipoclorito de sódio (2\%) por 3 minutos, e dispostas em um número de três sementes por tubete. Os tubetes foram previamente preenchidos com o substrato comercial Rohrbacher ${ }^{\circledR} \mathrm{e}$ as respectivas dosagens do adubo de liberação lenta. Os tubetes foram mantidos sob irrigação por microaspersores bailarina com vazão de $85 \mathrm{~L} \mathrm{~h}^{-1}$ em viveiro coberto com sombrite de $50 \%$. A temperatura e umidade relativa do ar média durante o período experimental foi, respectivamente, de $30,1^{\circ} \mathrm{C}$ e $51,3 \%$.

Aos 15 dias após semeadura (DAS) efetuou-se um primeiro raleio deixando-se duas plantas por tubete. Aos 30 DAS um segundo raleio foi realizado, deixando- se apenas uma muda por tubete.

A partir do $40^{\circ} \mathrm{DAS}$, as mudas receberam fertirrigação semanal, com $6 \mathrm{~mL}$ planta- ${ }^{1}$ de solução aquosa, contendo $4 \mathrm{~g} \mathrm{~L}^{-1}$ de sulfato de amônio, $10 \mathrm{~g} \mathrm{~L}^{-1}$ de superfosfato simples, $4 \mathrm{~g} \mathrm{~L}^{-1}$ de cloreto de potássio e $1 \mathrm{~g} \mathrm{~L}^{-1} \mathrm{de}$

Eq. (01):

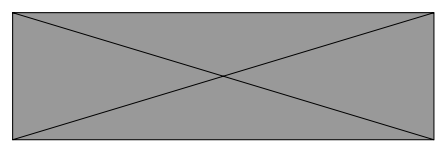

Análise estatística

Os resultados obtidos foram submetidos à análise de variância e ao ser constatada a significância pelo teste $\mathrm{F}$, as médias dos tratamentos foram comparadas pelo teste Tukey a $5 \%$ de probabilidade por meio do pacote ExpDes.pt (FERREIRA et al., 2013) do software livre R (R CORE TEAM, 2015). O efeito das doses de adubo de liberação controlada Osmocote ${ }^{\circledR}$ foi analisado por meio de regressões, sendo apresentadas somente as equações cujos coeficientes de maior grau foram significativos ( $p$ $<0,05)$.

\section{Resultados e Discussão}

Houve efeito significativo da interação entre os fatores avaliados (volumes de tubetes e doses de Osmocote $^{\circledR}$ ) para as variáveis altura, massa seca da parte aérea, massa seca de raiz, massa seca total e IQD nas mudas de baru aos 120 dias de idade (Figura 1).

Para as variáveis altura (Figura 1A), massa seca da parte aérea (MSPA) (Figura 1B), massa seca da raiz (MSR) (Figura 1C), massa seca total (MST) (Figura 1D) e IQD (Figura 1E) a resposta foi quadrática, apresentando um ponto de máxima eficiência técnica.

Para o tubete com volume de $55 \mathrm{~cm}^{3}$ só houve ajuste para variável altura, sendo possível observar que sua melhor média $(19,13 \mathrm{~cm})$ foi encontrada na dose 5,21 $\mathrm{g} \mathrm{dm}^{-3}$ (Figura 1A). O tubete de $280 \mathrm{~cm}^{3}$ proporcionou
FTE BR12 (9\% Zn, 3\% Fe, 2\% Mn, 0,1\% Mo, 1,8\% B, $0,8 \% \mathrm{Cu})$.

\section{Parâmetros avaliados}

Aos 120 dias após a semeadura foram avaliados a altura da parte aérea $(\mathrm{H} ; \mathrm{cm})$ e o diâmetro do coleto (DC; $\mathrm{mm}$ ) . A mensuração da altura das mudas foi realizada com o auxílio de uma régua milimetrada posicionada ao nível do solo até o meristema apical. O diâmetro foi medido por meio do uso de um paquímetro digital da marca Western, medido ao nível da borda do tubo.

Em seguida, as plantas foram colhidas e separadas em parte aérea e sistema radicular, lavadas em água corrente e secas em estufa com circulação forçada de ar, a aproximadamente $65^{\circ} \mathrm{C}$, até peso constante. Avaliou-se a massa seca da parte aérea (MSPA; $g$ planta ${ }^{-1}$ ), massa seca da raiz (MSR; $g$ planta $^{-1}$ ) e massa seca total (MST $=$ MSPA + MSR; g planta $\left.{ }^{-1}\right)$. Esses parâmetros foram transformados em índices de qualidade de mudas conforme sugerido por Gomes et al. (2002): H/DC, H/MSPA, MSPA/MSR e no Índice de Qualidade de Dickson - IQD (Dickson et al., 1960), calculado por:

as maiores médias para as variáveis altura $(25,49 \mathrm{~cm})$, MSPA (24,19 g planta $\left.^{-1}\right)$, MSR (16,18 $\mathrm{g}$ planta $\left.^{-1}\right)$, MST $\left(40,23 \mathrm{~g} \mathrm{planta}^{-1}\right)$ e IQD $(6,52)$ nas doses 4,$35 ; 5,29$; 4,67; 5,03 e 4,66 $\mathrm{g} \mathrm{dm}^{-3}$, respectivamente nas mudas de baru (Figura 1).

O tubete com volume de $180 \mathrm{~cm}^{3}$ apresentou médias inferiores ao volume de $280 \mathrm{~cm}^{3}$ para as variáveis altura, MSPA, MSR e MST (Figura 1). Sendo sua maior média para altura $\left(24,88 \mathrm{~cm}\right.$ ) na dose $5,54 \mathrm{~g} \mathrm{dm}^{-3}$ (Figura $1 \mathrm{~A})$, e o ponto máximo para MSPA $\left(16,17 \mathrm{~g} \mathrm{planta}^{-1}\right)$, MSR (11,74 g planta $\left.^{-1}\right)$ e MST (27,82 g planta-1) foram encontrados nas doses 3,68 $\mathrm{g} \mathrm{dm}^{-3}$ (Figura 1B); 3,27 $\mathrm{g}$ $\mathrm{dm}^{-3}$ (Figura 1C) e $3,50 \mathrm{~g} \mathrm{dm}^{-3}$ (Figura 1D), respectivamente.

Resultados semelhantes foram observados por Rossa et al. (2013). Os autores obtiveram respostas quadráticas no desempenho de mudas Sebastiania commersoniana ao avaliarem volumes de tubetes e doses de FLL, e concluíram que a dose mais eficiente foi de $5,54 \mathrm{~g} \mathrm{dm}^{-3}$ nos tubos de $180 \mathrm{~cm}^{3}$. Stupp et al. (2015) trabalhando com Mimosa scabrella observaram que as doses mais eficientes para o crescimento das plantas foram de 7,70 e 6,25 g $\mathrm{dm}^{-3}$ para os tubos de 110 e $180 \mathrm{~cm}^{3}$, respectivamente. Cabreira et al. (2019), evidenciaram que as mudas de Schizolobium parahyba responderam à fertilização até a dose $6 \mathrm{~g} \mathrm{dm}^{-3}$ para a variável altura nos tubos de 280 $\mathrm{cm}^{3}$. 
Desenvolvimento de mudas de baru em resposta a diferentes volumes de tubetes e doses de adubo de liberação lenta

O resultado encontrado no presente estudo, aliado aos citados anteriormente sugerem que as doses ideais de FFL podem variar conforme o recipiente e as espécies. Assim, o volume do recipiente e as doses de Osmocote ${ }^{\circledR}$ influenciam a qualidade das mudas, e isso se deve, possivelmente, ao fato desses elementos esta- rem diretamente relacionados com a disponibilidade de espaço físico e o aporte de nutrientes, que são condições essenciais para o cultivo de plantas ex situ.

Houve efeito significativo dos diferentes volumes de tubetes para as variáveis altura da parte aérea, MSPA, MSR, MST e IQD das mudas de baru (Tabela 2).

Figura 1 - Altura, massa seca da parte aérea, massa seca de raiz, massa seca total e Índice de Qualidade de Dickson (IQD) de mudas de baru aos 120 dias, em resposta a diferentes volumes de recipientes e doses de Osmocote ${ }^{\circledR}$

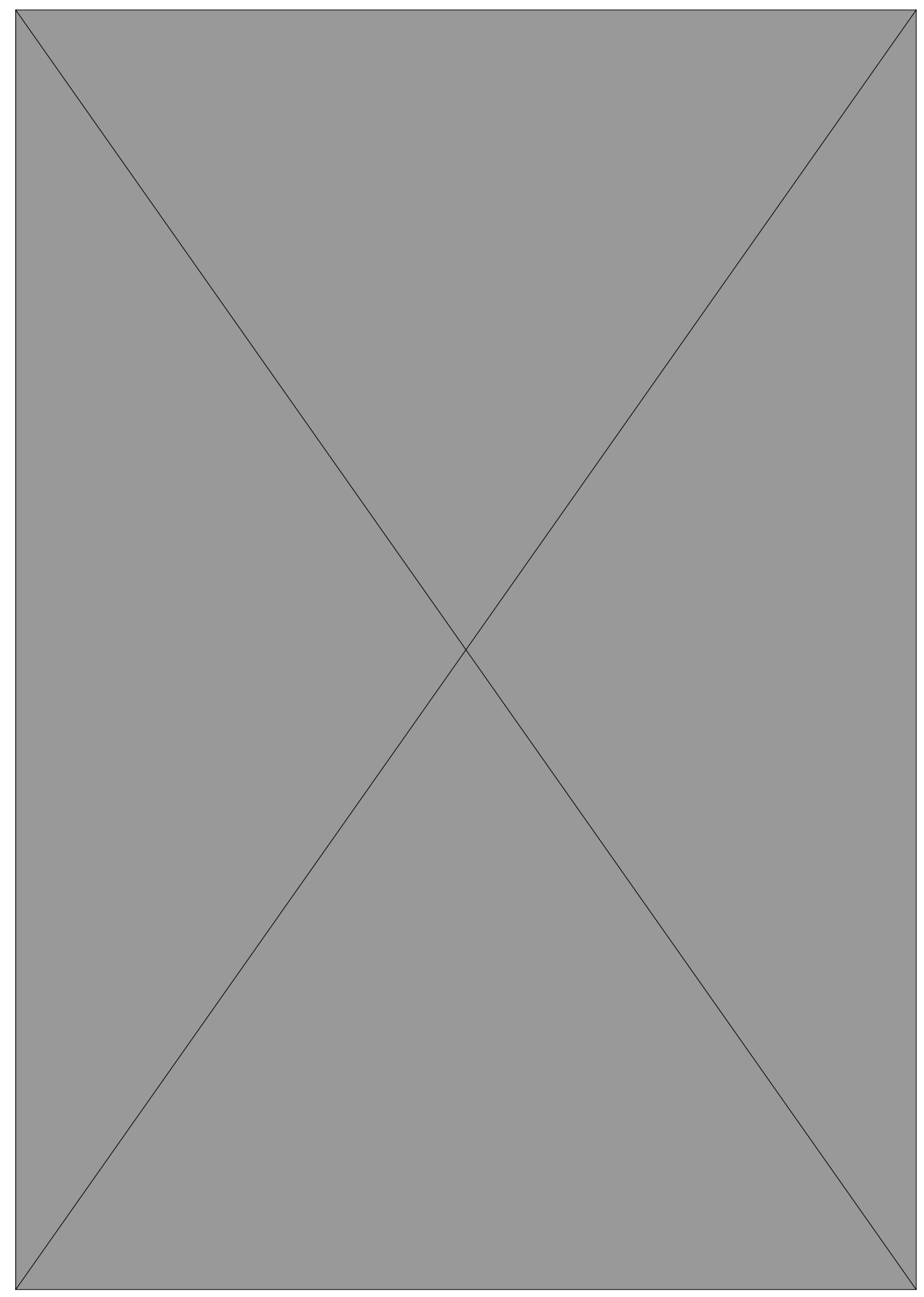

As maiores médias de altura da parte aérea foram observadas no volume de $280 \mathrm{~cm}^{3}$, entretanto, se igualando estatisticamente com o volume de $180 \mathrm{~cm}^{3}$ nas doses 9,0 e $12,0 \mathrm{~g} \mathrm{dm}^{-}{ }^{3}$. O volume de $55 \mathrm{~cm}^{3}$ apresentou os menores ganhos em altura nas mudas de baru. (Tabe- la 2). Esse resultado evidencia que as maiores taxas de crescimento foram encontradas nos tubetes com menores níveis de restrição ao sistema radicular, em que as mudas dispuseram de maior volume para desenvolver 
Aguilar, M. V. M. et al.

as raízes, refletindo-se no crescimento mais avantajado na parte aérea.

Gasparin et al. (2015), trabalhando com doses de Osmocote ${ }^{\circledR}$ e volumes de tubetes na produção de mudas de Parapiptadenia rigida (Benth.) Brenan, observou que o maior volume estudado, $180 \mathrm{~cm}^{3}$, apresentou as maiores médias para a variável altura.

Tabela 2 - Valores médios de altura da parte aérea, massa seca da parte aérea, massa seca da raiz, massa seca total e Índice de Qualidade de Dickson (IQD) de mudas de baru aos 120 dias, em resposta a diferentes volumes de tubetes

\begin{tabular}{|c|c|c|c|c|c|}
\hline \multirow{3}{*}{ Volume } & \multicolumn{5}{|c|}{ Altura da parte aérea $(\mathrm{cm})^{1}$} \\
\hline & \multicolumn{5}{|c|}{ Dose $\left(\mathrm{g} \mathrm{dm}^{-3}\right)$} \\
\hline & 0,0 & 3,0 & 6,0 & 9,0 & 12,0 \\
\hline $55 \mathrm{~cm}^{3}$ & $12,14 \mathrm{~b}$ & $13,85 \mathrm{c}$ & $16,23 \mathrm{c}$ & $20,48 \mathrm{~b}$ & $18,26 \mathrm{~b}$ \\
\hline $180 \mathrm{~cm}^{3}$ & $14,09 \mathrm{ab}$ & $16,28 \mathrm{~b}$ & $20,88 \mathrm{~b}$ & 25,55 a & $23,76 \mathrm{a}$ \\
\hline $280 \mathrm{~cm}^{3}$ & $16,13 \mathrm{a}$ & 18,79 a & 24,26 a & $26,52 \mathrm{a}$ & $24,44 \mathrm{a}$ \\
\hline \multirow{3}{*}{ Volume } & \multicolumn{5}{|c|}{ Massa seca da parte aérea $\left(g_{\text { planta }}^{-1}\right)^{1}$} \\
\hline & \multicolumn{5}{|c|}{ Dose $\left(\mathrm{g} \mathrm{dm}^{-3}\right)$} \\
\hline & 0,0 & 3,0 & 6,0 & 9,0 & 12,0 \\
\hline $55 \mathrm{~cm}^{3}$ & $6,74 \mathrm{a}$ & 8,83 a & $10,39 \mathrm{~b}$ & $10,46 \mathrm{c}$ & $9,23 \mathrm{~b}$ \\
\hline $180 \mathrm{~cm}^{3}$ & 7,65 a & 12,95 a & $14,54 \mathrm{ab}$ & $17,30 \mathrm{~b}$ & $13,60 \mathrm{~b}$ \\
\hline $280 \mathrm{~cm}^{3}$ & 9,49 a & 13,63 a & 17,33 a & 27,19 a & $22,28 \mathrm{a}$ \\
\hline \multirow{3}{*}{ Volume } & \multicolumn{5}{|c|}{ Massa seca da raiz $\left(g \text { planta }^{-1}\right)^{1}$} \\
\hline & \multicolumn{5}{|c|}{ Dose $\left(\mathrm{g} \mathrm{dm}^{-3}\right)$} \\
\hline & 0,0 & 3,0 & 6,0 & 9,0 & 12,0 \\
\hline $55 \mathrm{~cm}^{3}$ & 5,59 a & $6,86 b$ & $7,65 \mathrm{~b}$ & $7,03 \mathrm{c}$ & $6,79 b$ \\
\hline $180 \mathrm{~cm}^{3}$ & $7,02 \mathrm{a}$ & $10,38 \mathrm{ab}$ & 11,75 a & $11,04 \mathrm{~b}$ & $9,15 \mathrm{~b}$ \\
\hline \multirow[t]{2}{*}{$280 \mathrm{~cm}^{3}$} & $8,68 \mathrm{a}$ & $10,80 \mathrm{a}$ & $12,74 \mathrm{a}$ & 19,16 a & $14,75 \mathrm{a}$ \\
\hline & \multicolumn{5}{|c|}{ Massa seca total $\left(\mathrm{g} \text { planta }^{-1}\right)^{1}$} \\
\hline \multirow[t]{2}{*}{ Volume } & \multicolumn{5}{|c|}{ Dose $\left(\mathrm{g} \mathrm{dm}^{-3}\right)$} \\
\hline & 0,0 & 3,0 & 6,0 & 9,0 & 12,0 \\
\hline $55 \mathrm{~cm}^{3}$ & $12,33 \mathrm{a}$ & $15,69 \mathrm{~b}$ & $18,04 \mathrm{~b}$ & $17,49 \mathrm{c}$ & $16,03 \mathrm{~b}$ \\
\hline $180 \mathrm{~cm}^{3}$ & $14,67 \mathrm{a}$ & $23,33 \mathrm{ab}$ & $26,30 \mathrm{ab}$ & $28,34 \mathrm{~b}$ & $22,75 \mathrm{~b}$ \\
\hline $280 \mathrm{~cm}^{3}$ & $18,18 \mathrm{a}$ & $24,44 \mathrm{a}$ & 30,08 a & $46,35 \mathrm{a}$ & $37,03 \mathrm{a}$ \\
\hline \multirow{3}{*}{ Volume } & \multicolumn{5}{|c|}{ IQD $\left(g_{\text { planta }}^{-1}\right)^{1}$} \\
\hline & \multicolumn{5}{|c|}{ Dose $\left(\mathrm{g} \mathrm{dm}^{-3}\right)$} \\
\hline & 0,0 & 3,0 & 6,0 & 9,0 & 12,0 \\
\hline $55 \mathrm{~cm}^{3}$ & $2,93 \mathrm{a}$ & $3,36 \mathrm{~b}$ & $3,55 \mathrm{~b}$ & $2,77 \mathrm{c}$ & $2,86 \mathrm{~b}$ \\
\hline $180 \mathrm{~cm}^{3}$ & $3,57 \mathrm{a}$ & $4,91 \mathrm{a}$ & $4,68 \mathrm{ab}$ & $4,23 \mathrm{~b}$ & $3,59 \mathrm{~b}$ \\
\hline $280 \mathrm{~cm}^{3}$ & $4,04 \mathrm{a}$ & $5,10 \mathrm{a}$ & $5,11 \mathrm{a}$ & $7,61 \mathrm{a}$ & 6,04 a \\
\hline
\end{tabular}

${ }^{1}$ Médias seguidas da mesma letra minúscula na coluna não diferem entre si pelo teste Tukey a 5\% de probabilidade 
Desenvolvimento de mudas de baru em resposta a diferentes volumes de tubetes e doses de adubo de liberação lenta

O crescimento superior de espécies florestais em tubos plásticos de maiores volumes também foi relatado em outros estudos, como o de Ferraz e Engel (2011) com mudas de Hymenaea courbaril, Tabebuia chrysotricha e Parapiptadenia rigida, Stüpp et al. (2015) com Mimosa scabrella e por Figueiró et al. (2017), com mudas de Schizolobium parahyba.

A altura é uma característica relevante quando há a necessidade do plantio de mudas em áreas colonizadas por plantas espontâneas, onde a altura da espécie de interesse precisa exercer maior competição por luz. Dessa forma, a variável constitui um dos parâmetros importantes para estimar o crescimento no campo, visto que sua medição não causa destruição, apresenta fácil execução, e tecnicamente é aceita como uma excelente medida do potencial de desempenho das mudas (Rossa et al., 2015b).

Para as variáveis MSPA, MSR e MST observou-se que o volume de $280 \mathrm{~cm}^{3}$ proporcionou as melhores médias, se diferenciando estatisticamente dos demais volumes nas doses 9,0 e 12,0 $\mathrm{g} \mathrm{dm}^{-3}$ (Tabela 2).

Os volumes de recipientes testados interferiram no acúmulo de massa seca da parte aérea, sendo que o tubete de maior volume foi capaz de proporcionar excelentes ganhos médios, demonstrando assim, a sua grande viabilidade de uso na produção de mudas de baru. Esse resultado demonstra que o volume dos recipientes influencia a disponibilidade de nutrientes e água, devendo ser ressaltado que o maior volume promoveu maior produção de biomassa, e possivelmente, melhoria na arquitetura do sistema radicular.

O peso da massa seca da parte aérea indica rusticidade e essa variável correlaciona-se diretamente com a sobrevivência e desempenho inicial das mudas após o plantio em campo (Gomes e Paiva, 2006).

A biomassa total exerce papel significativo no desempenho das plantas quando transferidas para o campo, influenciando na capacidade de sustentação, absorção de água e nutrientes (Almeida et al., 2005).

Para o IQD foi possível observar que o volume de $280 \mathrm{~cm}^{3}$ apresentou as maiores médias em todas as doses, se diferindo estatisticamente dos demais tubetes de 55 e $180 \mathrm{~cm}^{3}$ nas doses 9,0 e $12 \mathrm{~g} \mathrm{dm}^{-3}$ (Tabela 2).

O Índice de Qualidade de Dickson é considerado promissor por considerar as associações entre os caracteres dendrométricos e alométricos em sua fórmula matemática, pois utiliza diversos parâmetros morfológicos relevantes (Eloy et al., 2013). Para seu cálculo são consideradas a robustez e o equilíbrio da distribuição da biomassa na muda, ponderando os resultados de várias características importantes empregadas para avaliação da qualidade das mudas (Fonseca et al., 2002). Portanto, quanto maior o IQD, melhor é a qualidade da muda produzida.

Não houve efeito significativo da interação entre o volume do tubete e doses de Osmocote ${ }^{\circledR}$ para a variável diâmetro do coleto e as relações H/DC e H/MSPA das mudas de baru, ocorrendo apenas efeitos isolados para esses fatores (Tabela 3).

Tabela 3 - Valores médios de diâmetro do coleto, relação altura da parte aérea e diâmetro do coleto (H/DC) e relação altura da parte aérea e massa seca da parte aérea (H/MSPA) de mudas de baru aos 120 dias, em resposta a diferentes volumes de tubetes

\begin{tabular}{cccc}
\hline Volume & $\begin{array}{c}\text { Diâmetro do coleto } \\
(\mathbf{m m})^{1}\end{array}$ & H/DC ${ }^{1}$ & H/MSPA $^{\mathbf{1}}$ \\
\hline $55 \mathrm{~cm}^{3}$ & $4,22 \mathrm{c}$ & $3,83 \mathrm{~b}$ & $1,80 \mathrm{~b}$ \\
$180 \mathrm{~cm}^{3}$ & $4,78 \mathrm{~b}$ & $4,13 \mathrm{a}$ & $1,57 \mathrm{ab}$ \\
$280 \mathrm{~cm}^{3}$ & $5,33 \mathrm{a}$ & $4,19 \mathrm{a}$ & $1,33 \mathrm{a}$ \\
\hline
\end{tabular}

${ }^{1}$ Médias seguidas da mesma letra minúscula na coluna não diferem entre si pelo teste Tukey a 5\% de probabilidade

Observou-se que o volume de $280 \mathrm{~cm}^{3}$ promoveu maior ganho em diâmetro do coleto para as mudas, diferindo estatisticamente dos demais volumes testados (Tabela 3). Mudas com diâmetro do coleto maior se sustentam melhor, apresentam maior resistência ao curvamento (tombamento), maior tolerância aos danos provocados por insetos e herbívoros, comparado às mudas de menor diâmetro do coleto (Ferraz e Engel, 2011).

Resultados semelhantes ao desse trabalho foram observados em mudas de canafístula (Peltophorum dubium Sprengel) (Massad et al., 2017), e paineira (Ceiba speciosa (A. St.-Hil.) Ravenna) (Lima Filho et al., 2019) com valores superiores para o diâmetro do coleto nas mudas em recipientes de maior volume. Rossa et al. (2015a) ressaltam que entre as variáveis avaliadas, o diâmetro do coleto é um parâmetro bastante propício para avaliar a qualidade de muda, devido ao seu maior grau de relação com o IQD.

Para a relação H/DC não houve diferença estatística entre o volume de 180 e $280 \mathrm{~cm}^{3}$ (Tabela 3). Esse resultado pode ser atribuído ao maior espaço ofertado pelos tubetes maiores, quando comparados ao volume 
Aguilar, M. V. M. et al.

de $55 \mathrm{~cm}^{3}$, consequentemente, possibilitando uma maior área de exploração com água e nutrientes pelo sistema radicular da muda, favorecendo seu desenvolvimento.

Foi possível observar que o tubete com volume de $280 \mathrm{~cm}^{3}$ proporcionou a menor média para a relação H/MSPA, mas não diferiu estatisticamente do volume de $180 \mathrm{~cm}^{3}$ (Tabela 3). Essa relação pode predizer o potencial de sobrevivência da muda no campo, uma vez que quanto menor for esse índice, mais lignificada está a muda e maior a sua rusticidade (Gomes et al., 2003). Em tubetes de $55 \mathrm{~cm}^{3}$ essa relação foi maior, indicando que possivelmente as mudas de baru produzidas nesse volume podem apresentar menor sobrevivência após o plantio no campo.

Os tubetes de menor volume restringem o crescimento do sistema radicular e, como as plantas tendem a ter um desenvolvimento equilibrado com a parte aérea, pode ocorrer uma interferência nos parâmetros morfológicos (Lisboa et al., 2012).

As relações $\mathrm{H} / \mathrm{DC}, \mathrm{H} / \mathrm{MSPA}$ e MSPA/MSR nas mudas de baru foram influenciadas significativamente pelas diferentes doses de Osmocote ${ }^{\circledR}$ (Figura 2).

Figura 2 - Relações H/DC, H/MSPA e MSPA/MSR de mudas de baru aos 120 dias, em resposta a diferentes doses de Osmocote $^{\circledR}$

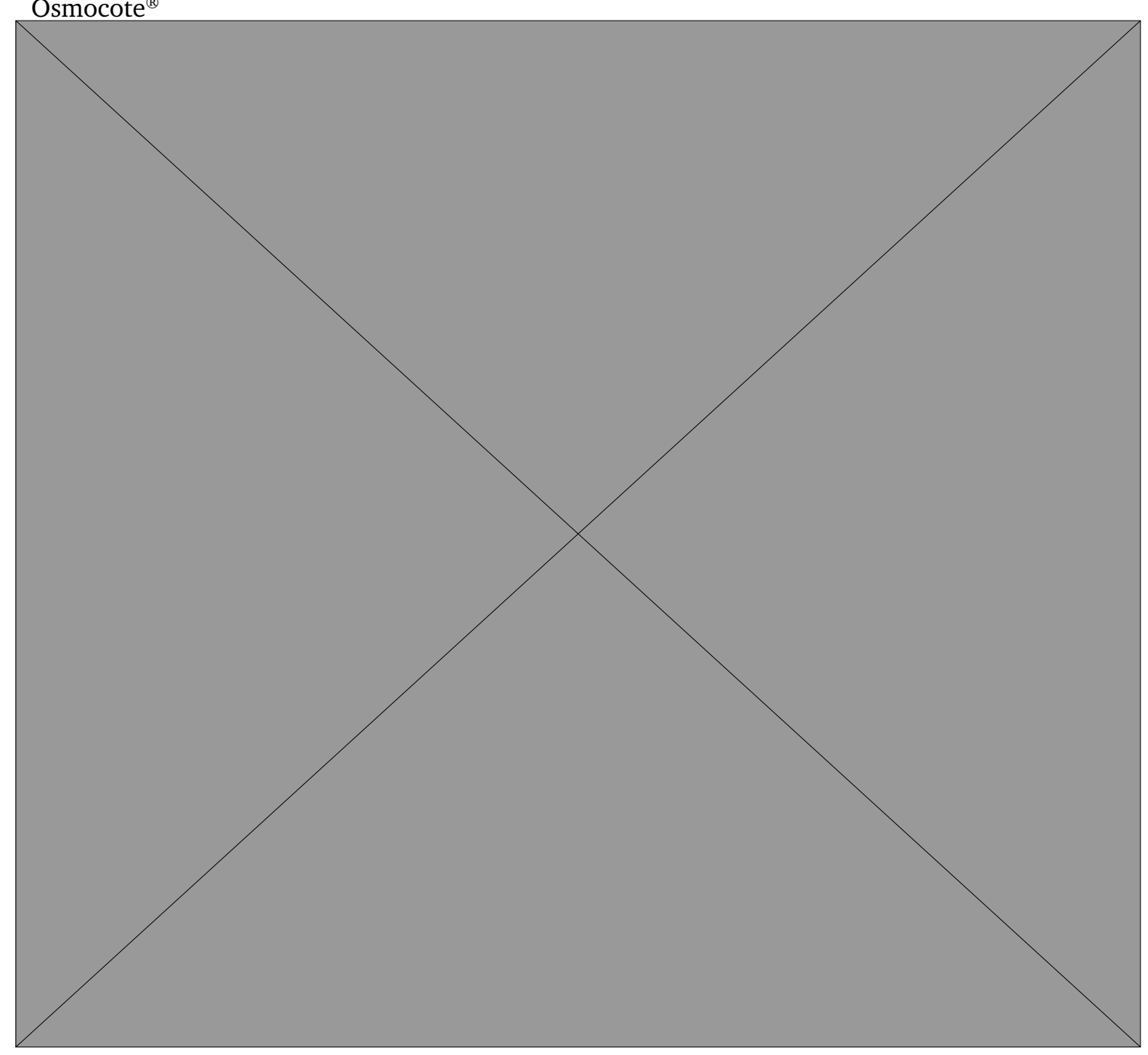

A relação H/DC, H/MSPA e MSPA/MSR também obtiveram resposta quadrática, apresentando um ponto de máxima eficiência técnica (Figura 2). A melhor resposta com a aplicação do fertilizante nas variáveis H/DC $(4,71)$, H/MSPA $(1,42)$ e MSPA/MSR $(1,48)$ foi nas doses de $5,10 \mathrm{~g} \mathrm{dm}^{-3} ; 3,15 \mathrm{~g} \mathrm{dm}^{-3}$ e 5,50 $\mathrm{g} \mathrm{dm}^{-3}$, respectivamente.

Resultados semelhantes foram observados por Rossa et al. (2011), com respostas quadráticas no desempenho de mudas de Ocotea odorífera, com as melhores médias para H/DC encontradas nas doses do FLL entre
5,43 e 6,39 $\mathrm{g} \mathrm{dm}^{-3}$. Dutra et al. (2016) ao avaliarem diferentes doses de Osmocote ${ }^{\circledR}$ em mudas de canafístula (Peltophorum dubium), concluíram que as mudas apresentaram melhor crescimento e padrão de qualidade sob doses entre 5,4 a 8,2 $\mathrm{g} \mathrm{dm}^{-3}$. Navroski et al. (2016) evidenciaram que as mudas de cedro (Cedrela fissilis) apresentam bom crescimento na dose de 5,0 g dm-3 de Osmocote $^{\circledR}$. Rosa et al. (2018) verificaram melhora no crescimento de plântulas de Moringa oleifera ao usar o fertilizante de liberação controlada na dose de 5,37 g $\mathrm{dm}^{-3}$ de substrato. 
Desenvolvimento de mudas de baru em resposta a diferentes volumes de tubetes e doses de adubo de liberação lenta

Essa tendência também foi relatada por Rossa et al. (2015a), que verificaram que a aplicação de fertilizante de liberação lenta resultou em ganhos significativos de crescimento para mudas de angico vermelho (Anadenanthera peregrina) e mudas de aroeira vermelha (Schinus terebinthifolius), com as melhores doses para o angico vermelho entre 5,54 e 6,68 $\mathrm{g} \mathrm{dm}^{-3}$.

A fertilização acima do nível máximo de eficiência técnica estimada pode provocar redução das médias das variáveis, como observado nas doses mais elevadas de Osmocote ${ }^{\circledR}$. Segundo Gasparin et al. (2015), isso se deve provavelmente, à toxicidade dos nutrientes, ou deficiências induzidas, como por exemplo, a competição pelo mesmo mecanismo de transporte entre íons com a mesma valência, inibindo a absorção de nutrientes.

Assim, doses baixas ou elevadas de Osmocote ${ }^{\circledR}$ causam um desequilíbrio nutricional nas mudas, prejudicando o crescimento delas no viveiro e podendo refletir na sua sobrevivência em campo, pós plantio. Esse fato reforça a importância de estudos particulares na busca por um melhor desenvolvimento das plantas e a otimização no uso da técnica de fertilização, sendo difícil a realização de uma recomendação padrão.

\section{Conclusão}

O tubete de $280 \mathrm{~cm}^{3}$ proporcionou maior crescimento inicial e produção de massa seca às mudas de Dipteryx alata (Vogel), conferindo um padrão de qualidade superior.

As mudas de baru responderam ao uso do adubo de liberação lenta Osmocote ${ }^{\circledR}$, apresentando melhores crescimento e padrão de qualidade sob doses entre 4,35 e $5,29 \mathrm{~g} \mathrm{dm}^{-3}$.

\section{Agradecimentos}

Ao Instituto Federal de Educação, Ciência e Tecnologia do Norte de Minas Gerais (IFNMG), Campus Salinas. À Coordenação de Aperfeiçoamento de Pessoal de Nível Superior - Brasil (CAPES) - Código de Financiamento 001.

\section{Referências}

Almeida, S. L.; Maia, N.; Ortega, A. R.; Angelo, A. C. 2005. Crescimento de mudas de Jacaranda puberula Cham. em viveiro submetidas a diferentes níveis de luminosidade. Ciência Florestal, 15: 323-329, 2005

Brito, L. P. S.; Bezerra, T. T.; Nunes, E. M. B.; Cavalcante, M. Z. B.; Siqueira Filho, J. A. 2018. Produção de mudas de Schinopsis brasiliensis Engler sob prévia lavagem do pó de coco e submetidas a doses crescentes de fertilizante de liberação controlada. Ciência Florestal, 28: 1022-1034.

Cabreira, G. V.; Leles, P. S. S.; Alonso, M.; Abreu, A. H. M.; Arthur Junior, J. C.; Vieira, A. V. G.; Lopes, N. F. 2019. Fertilization and containers in the seedlings production and post-planting survival of Schizolobium parahyba. Ciência Florestal, 29: 1644-1657. Doi: https:// doi.org/10.5902/1980509833261.

Carvalho, C. M.; Silva, C. R. 1992. Determinação das propriedades físicas de substrato. Botucatu: Faculdade de Ciências Agronômicas: Universidade Estadual Paulista.

Dias, G. P; Rodrigues, A. A.; Costa, A. M.; Carlos, L.; Vasconcelos Filho, S. V.; Batista, P. F. 2018. Morphological, anatomical and physiological characteristics of Acrocarpus fraxinifolius Wight e Arn seedlings according to containers and fertilization. Cerne, 24: 430-438.

Dickson, A.; Leaf, A. L.; F. Hosner, J. 1960. Qualitty appraisal of white spruce and white pine seedling stock in nurseries. Forestry Chronicle, 36: $10-13$.

Dutra, T. R.; Massad, M. D.; Sarmento, M. F. Q. 2016. Fertilizante de liberação lenta no crescimento e qualidade de mudas de canafístula (Peltophorum dubium). Revista Floresta, 46: 491-498.

Dutra, T. R.; Massad, M. D.; Sarmento, M. F. Q.; Oliveira, J. C. 2013. Substratos alternativos e métodos de quebra de dormência para produção de mudas de canafístula. Revista Ceres, 60: 72-78.

Eloy, E.; Caron, B. O.; Schmidt, D.; Behling, A.; Schwers, L. 2013. Avaliação da qualidade de mudas de Eucalyptus grandis utilizando parâmetros morfológicos. Revista Floresta, 43: 373-384.
EMBRAPA. Centro Nacional de Pesquisa de Solos, 1997. Manual de métodos de análise de solos. 2 ed. Rio de Janeiro: EMBRAPA.

Ferraz, A. V.; Engel, V. L. 2011. Efeito do tamanho de tubetes na qualidade de mudas de jatobá (Hymenaea courbaril L. Var. stilbocarpa (Hayne) Lee et Lang.), ipê-amarelo (Tabebuia chrysotricha (Mart. ex DC.) Sandl.) e guarucaia (Parapiptadenia rigida (Benth.) Brenan). Revista Árvore, 35: 413-423. Doi: https://doi.org/10.1590/S0100-67622011000300005.

Ferreira, E. B.; Cavalcanti, P. P; Nogueira, D. A. 2013. ExpDes.pt: experimental designs package $\mathrm{R}$ package version (1.1.2).

Figueiró, C. G.; Macedo, F. F; Fialho, L. F.; Silva, C. M. S.; Cândido, W. L. 2017. Efeito do recipiente e do método de superação de dormência no crescimento de mudas de Schizolobium parahyba (Vell.) S. F. Blake. Enciclopédia Biosfera, 14: 490-497.

Fonseca, E. P.; Valéri, S. V.; Miglioranza, E.; Fonseca, N. A. N.; Couto, L. 2002. Padrão de qualidade de mudas de Trema micrantha (L.) Blume, produzidas sobre diferentes períodos de sombreamento. Revista Árvore, 26: 515-523.

Freitas, E. C. S.; Paiva, H. N.; Leite, H. G.; Oliveira Neto, S. N. 2017. Crescimento e qualidade de mudas de Cassia grandis Linnaeus f. em resposta à adubação fosfatada e calagem. Ciência Florestal, 27: 509-519.

Garibay, S. L. C.; Aldrete, A.; López-Upton, J.; Ordáz-Chaparro, V. M. 2018. Efecto del envase, sustrato y fertilización en el crecimiento de Pinus greggii var. australis en vivero. Agrociencia, 52: 115-127.

Gasparin, E.; Araujo, M. M.; Saldanha, C. W.; Tolfo, C. V. 2015. Controlled release fertilizer and container volumes in the production of Parapiptadenia rigida (Benth.) Brenan seedlings. Acta Scientiarum. Agronomy, 37: 473-481. Doi: 10.4025/actasciagron.v37i4.19528.

Gibson, E. L.; Gonçalves, E. O.; Santos, A. R.; Araújo, E. F; Caldeira, M. V. W. 2019. Controlled-Release Fertilizer on Growth of Melanoxylon brauna Schott Seedlings. Floresta Ambiente, 26. 
Aguilar, M. V. M. et al.

Gomes, E. N.; Vieira, L. M.; Fagundes, C. M.; Rossa, U. B. Tofaneli, M. B. D. 2020. Controlled-release fertilizer increases growth, chlorophyll content and overall quality of loquat seedlings. Comunicata Scientiae, 11: 01-08. Doi: https://doi.org/10.14295/cs.v11i0.3353.

Gomes, J. M.; COUTO, L.; Garcia Leite, H. G.; Xavier, A.; Garcia, S. L. R. 2003. Crescimento de mudas de Eucalyptus grandis em diferentes tamanhos de tubetes e fertilização N-P-K. Revista Árvore, 23: 113-127. Disponível em: https://www.scielo.br/pdf/rarv/v27n2/15930.pdf.

Gomes, J. M. Couto, L.; Leite, H. G.; Xavier, A.; Garcia, S. L. R. 2002. Parâmetros morfológicos na avaliação da qualidade de mudas de Eucalyptus grandis. Revista Árvore, 26: 655-664.

Gomes, J. M.; Paiva, H. N. 2006. Viveiros Florestais: propagação sexuada. 3.ed. Viçosa: UFV.

Guimarães, R. A.; Miranda, K. M. C.; Chaves, L. J.; Naves, R. V.; Telles, M. P. C.; Soares, T. N. 2019. Mating system and pollen dispersal in Dipteryx alata Vogel (Leguminosae): comparing in situ and ex situ conditions. Tree Genetics \& Genomes, 15: 01-10.

Lima Filho, P.; Leles, P. S. S.; Abreu, A. H. M.; Fonseca, A. C.; Silva, E. V. 2019. Produção de mudas de Ceiba speciosa em diferentes volumes de tubetes utilizando o biossólido como substrato. Ciência Florestal, 29: 27-39. Doi: https://doi.org/10.5902/1980509819340.

Lisboa, A. C.; Santos, P. S.; Oliveira Neto, S. N.; Castro, D. N.; Abreu, A. H. M. 2012. Efeito do volume de tubetes na produção de mudas de Calophyllum brasiliense e Toona ciliata. Revista Árvore, 36: 603-609. Doi: https://doi.org/10.1590/S0100-67622012000400003.

Massad, M. D.; Dutra, T. R.; Meireles, I. E. S.; Sarmento, M. F. Q.; Santos, A. R.; Menezes, E. S. 2017. Avaliação do crescimento de canafístula em diferentes densidades de mudas por bandeja e volumes de tubetes. Ecologia e Nutrição Florestal, 5: 1-9, 2017.

Menegatti, R. D.; Guollo, K.; Navroski, M. C.; Vargas, O. F. 2017. Fertilizante de liberação lenta no desenvolvimento inicial de Aspidosperma parvifolium A. DC. Scientia Agraria Paranaensis, 16.

Navroski, M. C.; Tonett, E. L.; Mazzo, M. V.; Frigotto, T.; Pereira, M. O.; Galvani, L. V. 2016. Procedimento e adubação no crescimento inicial de mudas de cedro. Pesquisa Florestal Brasileira, 36: 17-24. Doi: 10.4336/2016.pfb.36.85.966.

R CORE TEAM. R: A language and environment for statistical computing. Vienna: R Foundation for Statistical Computing, 2015. Disponível em: http://www.R-project.org.

Rosa T. L. M.; Jordaim, R. B.; Alexandre, R. S.; Araujo, C. P.; Gonçalves, F. G.; Lopes, J. C. 2018. Controlled release fertilizer in the growth of Moringa oleifera LAM. seedlings. Floresta, 48: 303-310. Doi: 10.5380/ rf.v.48 i3.50063.
Rossa, U. B.; Angelo, A. C.; Nogueira, A. C.; Reissmann, C. B.; Grossi, F; Ramos, M. R. 2011. Fertilizante de liberação lenta no crescimento de mudas de Araucaria angustifolia e Ocotea odorifera. Revista Floresta, 41: 491-500. Doi: http://dx.doi.org/10.5380/rf.v41i3.24040.

Rossa, U. B.; Angelo, A. C.; Nogueira, A. C.; Westphalen, D. J.; Bassaco, A. V. M.; Milani, J. E. F.; Bianchin, J. E. 2013. Fertilizante de liberação lenta no desenvolvimento de mudas de Schinus terebinthifolius e Sebastiania commersoniana. Revista Floresta, 43: 93-104.

Rossa, U. B.; Angelo, A. C.; Westphalen, D. J.; Oliveira, F. E. M.; Silva, F. F. 2015a. Fertilizante de liberação lenta no desenvolvimento de mudas de Anadenanthera peregrina (L.) Speg. (angico-vermelho) e Schinus terebinthifolius Raddi (aroeira-vermelha). Ciência Florestal, 25: 841-852.

Rossa, U. B.; Angelo, A. C.; Bognola, I. A.; Westphalen, D. J.; Milani, J. E. 2015b. Fertilizante de liberação lenta no desenvolvimento de mudas de Eucalyptus grandis. Floresta, 45: 85-96.

Santos, A. R.: Gonçalves, E. O.; Gibson, E. L.; Araújo, E. F.; Caldeira, M. V. W. 2020. Controlled-release fertilizer in the growth of Dalbergia nigra seedlings. Floresta, 50: 1203-1212.

Santos, K. D. G.; Santos, K. D.; Ferreira, W. M.; Silva, K. L. F.; Coimbra, R. R.; Souza, C. C. 2018. Germination and initial development of Dipteryx alata Vogel (Fabaceae) in two "Cerrado" areas: a step toward its use in reforestation programs. Brazilian Journal of Botany.

Silva, F. B.; Costa, A. C.; Müller, C.; Nascimento, K. T.; Batista, P. F.; Vital, R. G.; Megguer, C. A.; Jakelaitis, A.; Domingos, M. 2020. Dipteryx alata, a tree native to the Brazilian Cerrado, is sensitive to the herbicide nicosulfuron. Ecotoxicology.

Silva, G. P.; Sales, J. F.; Nascimento, K. J. T.; Rodrigues, A. A.; Camelo, G. N.; Borges, E. E. D. L. 2020. Borgesd Biochemical and physiological changes in Dipteryx alata Vog. seeds during germination and accelerated aging. South African Journal of Botany, 131: 84-92.

Sousa, F. F.; Sousa, F. F.; Braga, R. M.; Venturin, N.; Macedo, R. L. G.; Carlos, L.; Venturin, R. P. 2018. Exigências nutricionais de mudas de Dipteryx alata sob limitação nutricional. Ciência Florestal, 28: 102-114. Doi: http://dx.doi.org/10.5902/1980509831586.

Stüpp, A. M.; Navroski, M. C.; Felippe, D.; Kniess, D. D. C.; Amâncio, J. C.; Silva, M. A.; Pereira, M. O. 2015. Crescimento de mudas de Mimosa scabrella Benth em função de diferentes tamanhos de recipientes e doses de fertilizante. Revista Ecologia e Nutrição Florestal, 3: 40-47.

Toledo, L. O.; Anjos, L. H. C.; Couto, W. H.; Correia, J. R.; Pereira, M. G.; Correia, M. E. F. 2009. Análise multivariada de atributos pedológicos e fitossociológicos aplicada na caracterização de ambientes de cerrado no norte de Minas Gerais. Revista Árvore, 33: 957-968. 